\title{
Defects controlled wrinkling and topological design in graphene
}

\author{
Zhang, Teng, teng_zhang@brown.edu; Gao, Huajin; Li, Xiaoyan, Brown University, United States
}

\begin{abstract}
Because of its atomic scale thickness, the deformation energy in a free standing graphene sheet can be easily released through out-of-plane wrinkles which, if controllable, may be used to tune the electrical and mechanical properties of graphene. Here we adopt a generalized von Karman equation for a flexible solid membrane to describe graphene wrinkling induced by a prescribed distribution of topological defects such as disclinations (heptagons or pentagons) and dislocations (heptagon-pentagon dipoles). In this framework, a given distribution of topological defects in a graphene sheet is represented as an eigenstrain field which is determined from a Poisson equation and can be conveniently implemented in finite element method simulations. Comparison with atomistic simulations indicates that the proposed model, with only three parameters (i.e., bond length, stretching modulus, and bending stiffness), is capable of accurately predicting the atomic scale wrinkles near disclination/dislocation cores while also capturing the large-scale graphene configurations under specific defect distributions such as those leading to a sinusoidal surface ruga or a catenoid funnel.
\end{abstract}

